\title{
Tracing the spiral arms in IP Pegasi
}

\author{
R. Baptista ${ }^{1}$, L. Morales-Rueda ${ }^{2}$, E. T. Harlaftis ${ }^{3, \star}$, T. R. Marsh ${ }^{4}$, and D. Steeghs ${ }^{5}$
}

${ }^{1}$ Departamento de Física, Universidade Federal de Santa Catarina, Campus Trindade, 88040-900, Florianópolis, SC, Brazil e-mail: bap@astro.ufsc.br

2 Department of Astrophysics, Radboud University Nijmegen, 6500 GL Nijmegen, The Netherlands e-mail: lmr@astro.kun.nl

3 Institute of Space Applications and Remote Sensing, National Observatory of Athens, PO Box 20048, Athens 118 10, Greece

4 Department of Physics, University of Warwick, Coventry CV4 7AL, UK e-mail: T.R.Marsh@warwick.ac.uk

5 High Energy Astrophysics Division, Center for Astrophysics, MS-67, 60 Garden Street, Cambridge, MA 02138, USA e-mail: dsteeghs@head.cfa.harvard.edu

Received 11 May 2005 / Accepted 1 July 2005

\section{ABSTRACT}

We report the analysis of time-resolved spectroscopy of IP Pegasi in outburst with eclipse mapping techniques to investigate the location and geometry of the observed spiral structures. We were able to obtain an improved view of the spiral structures with the aid of light curves extracted in velocity bins matching the observed range of velocities of the spiral arms combined with a double default map tailored for reconstruction of asymmetric structures. Two-armed spiral structures are clearly seen in all eclipse maps. The arms are located at different distances from the disc centre. The "blue" arm is farther out in the disc $\left(R=0.55 \pm 0.05 R_{L 1}\right)$ than the "red" $\operatorname{arm}\left(R=0.30 \pm 0.05 R_{L 1}\right)$. There is evidence that the velocity of the emitting gas along the spiral pattern is lower than the Keplerian velocity for the same disc radius. The discrepancy is smaller in the outer arm (measured velocities 10-15 per cent lower than Keplerian) and is more significant in the inner arm (observed velocities up to 40 per cent lower than Keplerian). We measured the opening angle of the spirals from the azimuthal intensity distribution of the eclipse maps to be $\phi=25^{\circ} \pm 3^{\circ}$. A comparison with similar measurements on data at different outburst stages reveals that the opening angle of the spiral arms in IP Peg decreases while the outbursting accretion disc cools and shrinks, in agreement with the expected evolution of a tidally driven spiral wave. The sub-Keplerian velocities along the spiral pattern and the clear correlation between the opening angle of the spirals and the outburst stage favors the interpretation of these asymmetric structures as tidally-induced spiral shocks.

Key words. stars: novae, cataclysmic variables - stars: individual: IP Pegasi - accretion, accretion disks - shock waves line: formation - techniques: spectroscopic

\section{Introduction}

IP Pegasi is a dwarf nova, a compact binary in which a late-type star overfills its Roche lobe and transfers matter to a companion white dwarf via an accretion disc. It shows recurrent outbursts (every 70-100 days) in which the disc expands and brightens by 2-3 mag during 10-12 days as a consequence of a sudden increase in mass accretion through the disc. In the quiescent, low-mass accretion state, the white dwarf and the bright spot (formed by the impact of the infalling gas stream with the outer edge of the disc) dominate the light from the binary at optical and ultraviolet wavelengths, whereas during outburst the hot and large disc is the dominant source of light. The binary is seen at a high inclination angle $\left(i=81^{\circ}\right)$, which leads to deep eclipses in the light curve every $3.8 \mathrm{~h}$ when the white dwarf, accretion disc and bright spot are occulted by the mass-donor star. This allows the emission from the different light sources to

* In memoriam. be distinguished and spatially resolved studies to be performed, making IP Peg an ideal laboratory for the study of accretion physics. In particular, it is well suited for the application of indirect imaging techniques to resolve the disc emission both in position (eclipse mapping, Horne 1985) and velocity (Doppler tomography, Marsh \& Horne 1988).

Additional attention has been drawn to IP Peg after the discovery of a two-armed spiral structure in its accretion disc during outburst (Steeghs et al. 1997). These spiral structures were seen in different outbursts and at different outburst stages from the end of the rise and maximum (Steeghs et al. 1997; Harlaftis et al. 1999; Baptista et al. 2000) through the decline stages (Morales-Rueda et al. 2000; Baptista et al. 2002) and possibly also at the very late decline (Saito et al. 2005). They are believed to be raised by tides from the mass-donor star on the outer accretion disc when it expands during outburst (see review by Steeghs 2001).

Spiral shocks in accretion discs were predicted theoretically and advocated as an alternative mechanism for the 
removal of angular momentum required for the disc gas to accrete (Sawada et al. 1986). Numerical hydrodynamic simulations suggest that strong spiral shocks are formed in hot accretion discs (e.g., Sato et al. 2003, and references therein) and that the opening angle of the spirals (the angle formed by the shock front and the azimuthal direction of the disc gas flow) depends on the disc temperature: the cooler the disc, the more tightly wound the spiral pattern is. Godon et al. (1998) argued that wide open spirals could only form in accretion discs much hotter $\left(T \sim 10^{6} \mathrm{~K}\right)$ than those of dwarf novae. Steeghs \& Stehle (1999) remarked that the disc radius is also important in determining the shape of the spirals and that wide open spiral arms such as those observed in IP Peg could be raised at the much lower temperatures found in accretion discs of outbursting dwarf novae $\left(T \simeq 10^{4} \mathrm{~K}\right)$ if the disc is large - as expected for outbursting discs.

In a previous paper (Harlaftis et al. 2004) we investigated the ability of the eclipse mapping method to recover spiral structures and we set the observational requirements for a successful reconstruction. We also showed how the detection of spiral arms with eclipse mapping is improved with a proper extraction of velocity-resolved eclipse light curves to avoid contamination by light sources other than the spiral arms. In this follow up paper we reanalyze the spectroscopic data of Morales-Rueda et al. (2000) with eclipse mapping techniques to investigate the spatial properties of the observed two-armed spiral structure.

This paper is organized as follows. The details of the analysis are presented in Sect. 2 while the results are presented and discussed in Sect. 3. Section 4 compares the orientation of the spiral arms from this study with those from the previous analysis of Baptista et al. (2000, hereafter BHS) and Baptista et al. (2002, hereafter BHT) to infer the changes in the opening angle of the spiral arms with outburst stage, and discusses the implications of our results for the interpretation of the observed spiral structures in terms of irradiation of tidally-thickened outer disc regions by a hot inner disc. The conclusions are summarized in Sect. 5. The Appendix discusses the changes implemented in the eclipse mapping method in order to allow an improved reconstruction of asymmetric structures such as spiral arms in accretion discs.

\section{Data analysis}

\subsection{Observations}

Time-resolved spectrophotometry of IP Pegasi was secured with the Intermediate Dispersion Spectrograph on the $2.5 \mathrm{~m}$ Isaac Newton Telescope, at La Palma, during the nights of 1994 October 30 and 31 . The observations were made 5 and 6 days after the onset of an outburst, when the star was at the maximum brightness plateau phase of the 12-14 day long outburst (Morales-Rueda et al. 2000, see their Fig. 1). A total of 164 blue spectra ( $\lambda \lambda$ 4040-4983 $⿱$ ) was obtained at a time resolution of $220 \mathrm{~s}$ and a spectral resolution of $100 \mathrm{~km} \mathrm{~s}^{-1}$ at $\mathrm{H} \beta$. The reader is referred to Morales-Rueda et al. (2000) for a detailed description of the data set and of the reduction procedures.
$\mathrm{H} \beta$

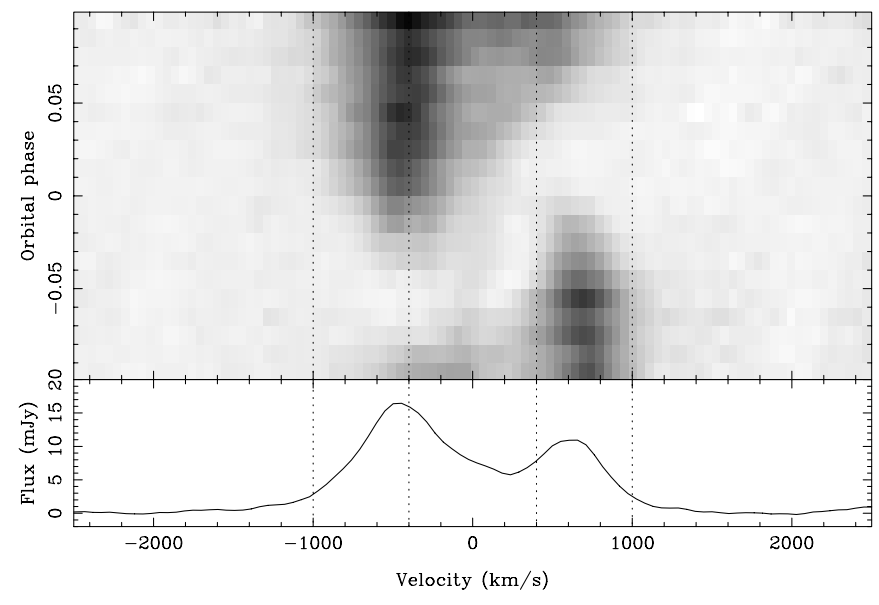

Fig. 1. Top: zoomed trailed spectrogram of the $\mathrm{H} \beta$ line around eclipse. Bright regions are dark. Bottom: the integrated line profile along the eclipse phases of the spectrogram on the top panel (from $\phi=-0.1$ to +0.1 cycle). Vertical dotted lines mark the two velocity-resolved regions used to extract light curves of the spiral arms and to isolate low-velocity emitting gas.

The spectra were phase-folded according to the linear ephemeris of Wolf et al. (1993),

$T_{\text {mid }}(\mathrm{HJD})=2445615.4156+0.15820616 E$

where $T_{\text {mid }}$ is the inferior conjunction of the secondary star. Our observations are bracketed by two sets of HST observations of IP Peg in quiescence (Baptista et al. 1994) in which the white dwarf eclipse egress times can be precisely measured. From these timings, we infer that the white dwarf mid-eclipse at that epoch occurred 0.008 orbital cycles before the prediction of the ephemeris in Eq. (1) (assuming a white dwarf eclipse width of $\Delta \phi=0.086$ cycles, Wood \& Crawford 1986). Therefore, an offset of +0.008 cycles was added to the phases in the light curve to make phase zero coincident with the inferior conjunction of the secondary star. The observations cover the eclipse cycles $E=25158,25159,25164$ and 25165 .

\subsection{Emission-line light curves and eclipse mapping}

The upper panel of Fig. 1 shows a zoomed trailed spectrogram of $\mathrm{H} \beta$ during eclipse (phase range -0.1 to +0.1 cycles) on the first night of observations. The lower panel shows the resulting line profile integrated over the eclipse phase range. The emission lines of IP Peg in outburst have contributions from the spiral arms as well as the irradiated face of the secondary star (Harlaftis 1999) and possibly another low-velocity emission component (Harlaftis et al. 1999; Steeghs et al. 1996). The signature of the two-armed spiral structure appears in Fig. 1 as the two bright arcs running from positive, high velocities $\left(v \geq 400 \mathrm{~km} \mathrm{~s}^{-1}\right.$ ) before mid-eclipse to negative, high velocities after phase zero. The observed change in line profile during eclipse indicates that the "red" spiral arm is being eclipsed at about the same phase $(\phi \sim-0.01$ cycles $)$ at which the "blue" arm starts reappearing from eclipse. The low-velocity emission 


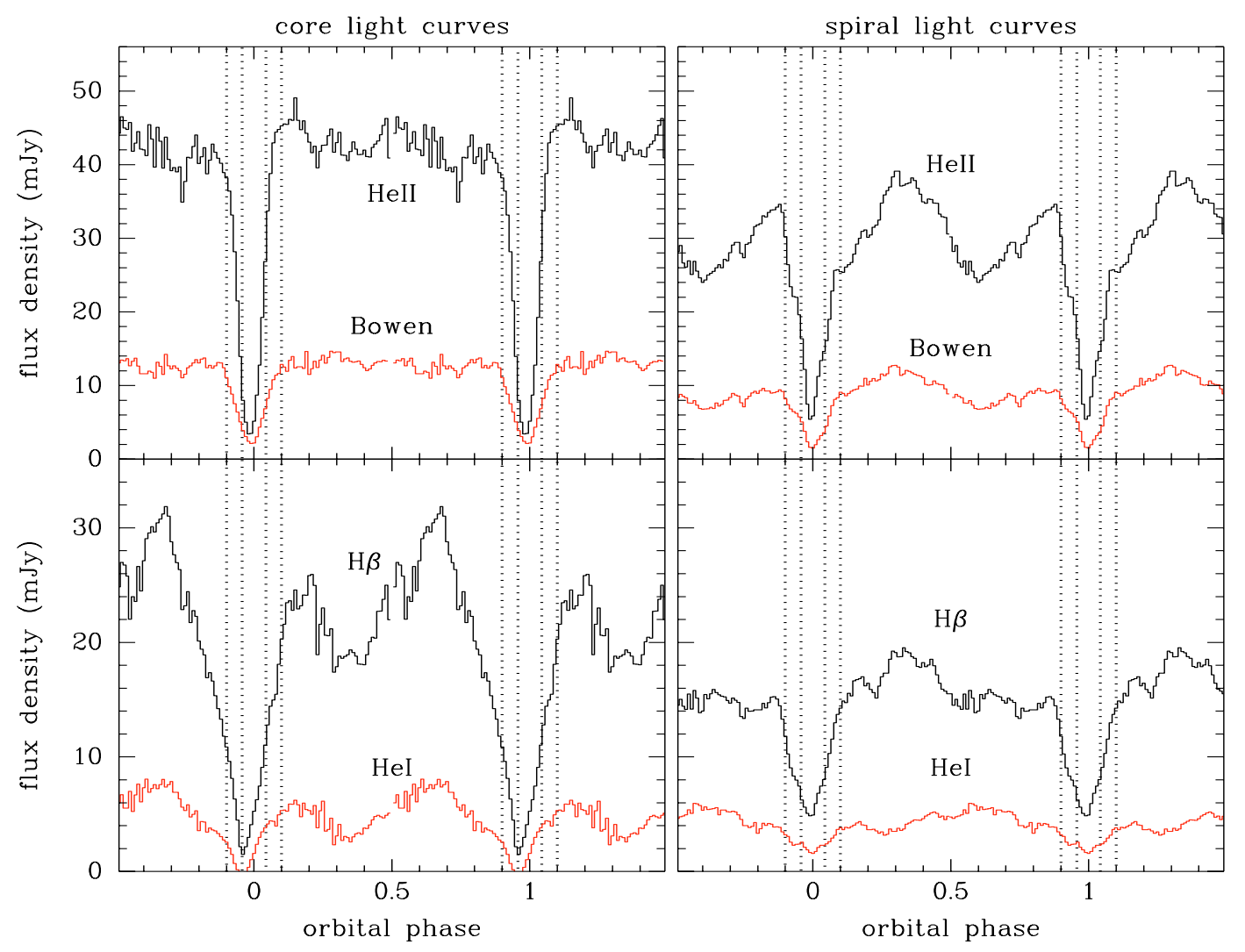

Fig. 2. Extracted core (left panels) and spiral (right panels) light curves for He II and H $\beta$ (in black) and for He I and the Bowen blend (in grey). Vertical dotted lines mark mid-ingress/egress phases of the white dwarf and the reference phases -0.1 and +0.1 cycles. The light curves are repeated in phase for visualization purposes. The $\mathrm{H} \gamma$ light curves (not shown) are very similar to those of $\mathrm{H} \beta$.

is seen as the faint line-emission component changing from blue to red shifts (at $v \leq 400 \mathrm{~km} \mathrm{~s}^{-1}$ ) around mid-eclipse.

Because the spiral arms and the other emission components contribute to the line profile at different Doppler velocities, it is possible to separate the contribution of these distinct light sources by a proper extraction of velocity-resolved eclipse light curves. For example, BHT performed velocity-resolved eclipse mapping to separate the HeI emission from each of the spiral arms in IP Peg. Harlaftis et al. (2004) discussed how dilution by other light sources reduces the ability of the eclipse mapping method to reconstruct spiral structures in accretion discs. They also showed how the detection of spiral arms may be improved with the aid of velocity-resolved light curves. Here we use this technique as an useful tool to minimize the contribution of the low-velocity gas to the light curve and to optimize the detection of the spiral arms in line eclipse maps.

Figure 1 shows the choice of the velocity-resolved passbands used to separate the contribution of the spirals from that of low-velocity gas in the emission lines of IP Peg. Two light curves were extracted for each line, one covering the low velocity range $\left(-400\right.$ to $\left.+400 \mathrm{~km} \mathrm{~s}^{-1}\right)$ and one matching the observed velocity range of the spiral arms $(-1000$ to -400 plus +400 to $+1000 \mathrm{~km} \mathrm{~s}^{-1}$ for all lines except He I 4472, for which we use the narrower ranges -900 to -400 plus +400 to $+900 \mathrm{~km} \mathrm{~s}^{-1}$ to avoid contamination by the $\mathrm{Mg}$ II $\lambda 4481 \AA$ line). The low-velocity range light curve will be hereafter referred to as the "core" light curve, while the higher velocity range curve will be referred to as the "spiral" light curve. Core and spiral light curves were extracted for $\mathrm{H} \beta, \mathrm{H} \gamma$, He II 4686, He I 4472 and the Bowen blend at $\lambda \lambda$ 4640-4660 $\AA$.

Four orbital cycles were observed. The orbital modulation and the shape of the eclipse are consistent from one night to the other (see Fig. 5 of Morales-Rueda et al. 2000), indicating that the disc brightness distribution is relatively stable over that time scale. We therefore combined the 4 light curves to produce median light curves at a phase resolution of 0.01 cycles for all lines. The error bars were computed from the absolute deviation with respect to the median in each phase bin. The resulting light curves are shown in Fig. 2.

The He II and Bowen blend core light curves show a narrow, deep and reasonably well centered eclipse indicating that the low-velocity emission in these lines arise from a region close to the white dwarf at disc centre. A possible interpretation would be that this line component is produced in a collimated, hot wind from the inner disc, in which most of the velocity is directed away from the orbital plane giving rise to small Doppler shifts. The Balmer and He I core light curves show the very broad, asymmetric and phase offset eclipse seen in Harlaftis et al. (2004, see their Figs. 7 and 8). The eclipse is too wide to be caused by occultation of the accretion disc by the secondary star. The shape of this eclipse is possibly produced by self-occultation of an asymmetric brightness distribution in the irradiated face of the secondary star or by 

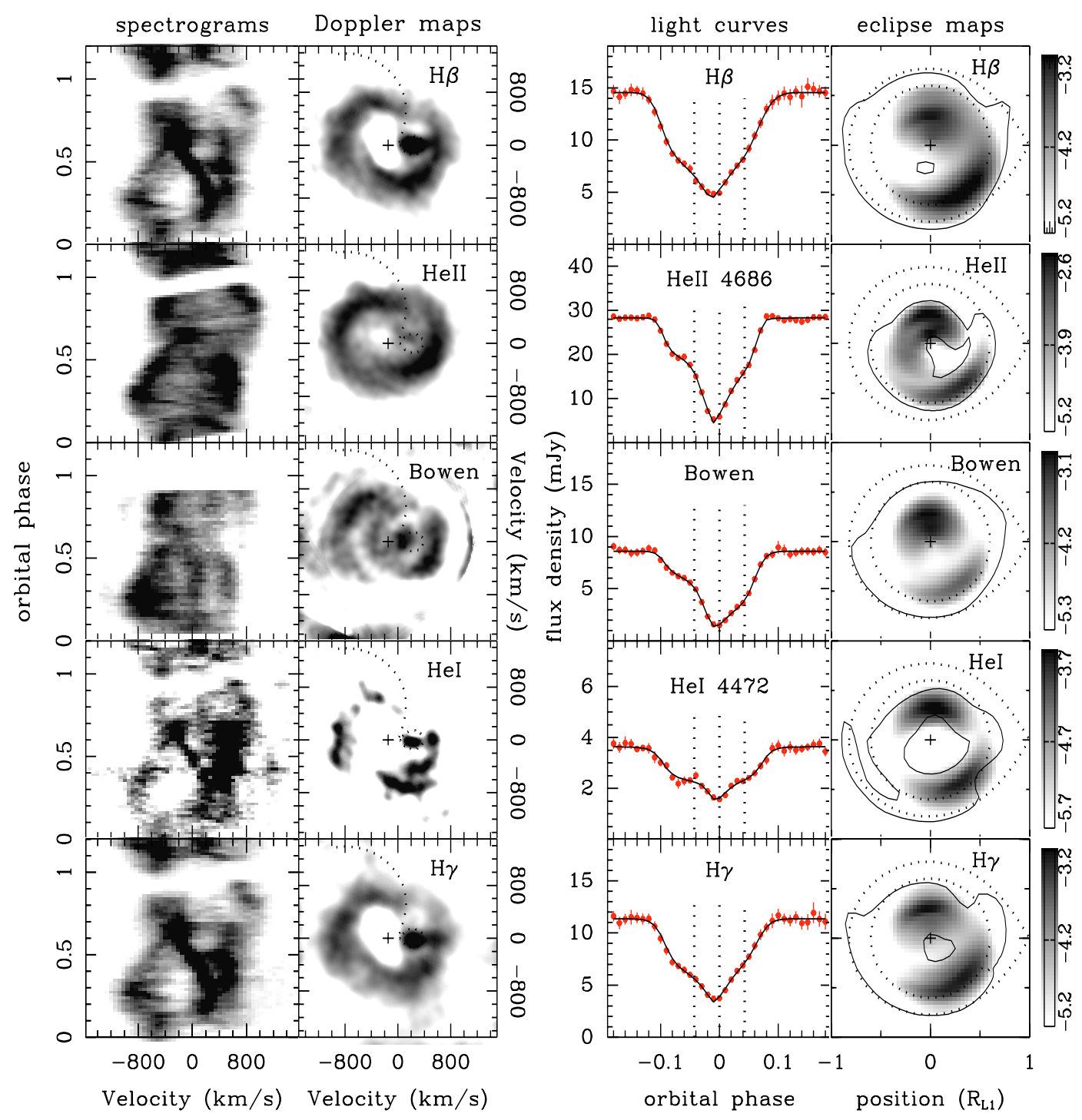

Fig. 3. Left: data trailed spectrograms (linear greyscale) and corresponding Doppler maps (logarithmic greyscale) for $\mathrm{H} \beta$, He II 4686 , the Bowen blend, He I 4472, and $\mathrm{H} \gamma$. Dark regions are brighter. A cross marks the position of the white dwarf. Dotted lines outline the Roche lobe of the secondary star and the velocities along the ballistic stream trajectory. Right: line spiral light curves and corresponding eclipse maps on a logarithmic greyscale. Dark regions are brighter. The bar in the right side indicates the greyscale used in each case. Dotted lines show the primary Roche lobe and a circle of radius $0.6 R_{L 1}$. A cross marks the centre of the disc. The secondary is to the right of each panel and the stars rotate counter-clockwise. A solid contour line is overploted on each eclipse map to indicate the 3- $\sigma$ confidence level region.

occultation of an emission source at a different position in the binary (e.g., Steeghs et al. 1996). On the other hand, all spiral light curves show the orbital modulation and asymmetric eclipse shape characteristics of the emission from a two-armed spiral structure in the accretion disc (e.g., BHS, Steeghs \& Stehle 1999). Two separate brightness sources are eclipsed in sequence. The first one is eclipsed at $\phi_{1 i} \simeq-0.09$ cycle and the second one at $\phi_{2 i} \simeq-0.02$ cycle; they reappear from eclipse at phases $\phi_{1 e} \simeq+0.02$ cycle and $\phi_{2 e} \simeq+0.07$ cycle, respectively (see Fig. 3 for a zoomed view of the light curves around eclipse). Hence, our choice of passband successfully framed the emission from the spiral arms in the spiral light curves ${ }^{1}$.

\footnotetext{
${ }^{1}$ Similar spiral light curves and eclipse shapes are obtained if we extend the spiral passband to higher velocity ranges (up to $|v|=$ $1300 \mathrm{~km} \mathrm{~s}^{-1}$, see Harlaftis et al. 2004). However, the resulting light
}

Morales-Rueda et al. (2000) found that the eclipses of the emission lines were significantly shifted towards negative phases. The +0.008 cycle offset applied to the data (Sect. 2.1) eliminates the phase offset of the continuum light curve and reduces the discrepancy for the line light curves. Figure 2 shows that the remaining phase offset is caused by asymmetries in the brightness distribution of the low-velocity line emitting gas (core light curves). There is no phase offset in the spiral light curves. Therefore, the shifted eclipses seen in the full-line light curves are not an evidence of unequal brightnesses in the two arms, as claimed by Smak (2001).

In the remainder of this paper we concentrate on the analysis of the spiral light curves. Our eclipse mapping code

curves have lower signal-to-noise ratios as a consequence of the reduced average flux. 
(Baptista \& Steiner 1993) assumes that any brightness change in the light curve is caused by occultation of disc regions by the secondary star. Therefore, the out-of-eclipse orbital modulation of the spiral light curves was removed by fitting a spline function to the regions of the light curve excluding the eclipse, dividing the light curve by the fitted spline, and scaling the result to the flux level of the spline function at phase zero (e.g., BHS). This procedure allows the removal of orbital modulations with only minor effects of the eclipse shape itself.

The eclipse maps are flat square arrays of $51 \times 51$ pixels and side $2 R_{L 1}$ centered on the white dwarf, where $R_{L 1}$ is the distance from disc centre to the inner Lagrangian point. The adopted eclipse geometry is $i=81^{\circ}$ and $q=0.5$ (Wood \& Crawford 1986), which ensures that the white dwarf is at the centre of the map. The reconstructions were obtained using a double default function with $\Delta \theta=10^{\circ}$ (see Appendix).

The statistical uncertainties of the eclipse maps were computed with a Monte Carlo procedure (e.g., Rutten et al. 1992). For each line light curve, we create a set of 20 artificial light curves in which the flux at each phase is varied around the true value according to a Gaussian distribution with standard deviation equals to the uncertainty at that point. These light curves are fitted with the eclipse mapping code to generate a set of randomized eclipse maps. These are combined to produce an average map and a map of the standard deviations with respect to the average. A map of the statistical significance (or the inverse of the relative error) is obtained by dividing the true eclipse map by the map of the standard deviations.

\section{Results}

The resulting eclipse maps are shown in Fig. 3 in a logarithmic grey-scale. For completeness, the data trailed spectrograms and the computed Doppler maps of the corresponding lines (Morales-Rueda et al. 2000) are shown in the left two columns. Doppler maps for the $\mathrm{H} \gamma$ and Bowen blend lines (not shown in Morales-Rueda et al. 2000) are also included. The Doppler maps and trailed spectrograms correspond to the data of the first night of observations, except for the Bowen blend map. In this case the data of the two nights were combined and the spectra around eclipse excluded from the analysis in order to produce a single Doppler map of better signal-to-noise ratio. The Doppler maps of Fig. 3 are shown rotated 90 degrees clockwise with respect to their usual presentation (e.g., Morales-Rueda et al. 2000) in order to display the spiral arms in a similar orientation as in the eclipse maps. The blue spiral arm appears closer to the secondary star in the lower right side of the Doppler map. We shall remark that the coordinate frames of the Doppler and eclipse maps are very different: the inner, low-velocity side of a spiral arm in the Doppler map is related to the outer side of the corresponding structure in the eclipse map.

The advantage of using velocity-resolved light curves matched to the observed velocity range of the spirals becomes clear when we compare our eclipse maps with those obtained from light curves over the full line width (BHS; Harlaftis et al. 2004). BHS needed to compute the asymmetric part of their eclipse maps in order to see clearly the spiral structures, whereas the significant dilution by other line emitting sources hampered the detection of the spiral arms in the Balmer and He I full-line eclipse maps of Harlaftis et al. (2004). Here, the spiral structures are conspicuous in the eclipse maps without the need of further data processing.

The double-stepped bulges in the eclipse shapes map into two asymmetric structures corresponding to the two-armed spiral structure seen in the Doppler maps. The solid contour line overploted on each eclipse map depicts the 3- $\sigma$ confidence level region as derived from the map of the statistical significance in each case (Sect. 2.2). The two-armed spiral structure is at or above the 3- $\sigma$ confidence level in all line maps. We will hereafter refer to these structures as to the "blue" and "red" spiral arms. They are located at different distances from disc centre. The red spiral arm is seen in the upper side of the eclipse map, at radii of $0.2-0.4 R_{L 1}$. The blue arm is seen in the lower right side of the map, at radii of $0.45-0.65 R_{L 1}$. This is in agreement with the Doppler tomography, which indicates smaller velocities for the blue spiral arm $\left(\simeq 600 \mathrm{~km} \mathrm{~s}^{-1}\right)$ than for the red spiral arm $\left(\sim 700 \mathrm{~km} \mathrm{~s}^{-1}\right)$ (Morales-Rueda et al. 2000). The red arm is brighter than the blue arm in the Bowen blend, whereas the opposite holds for $\mathrm{H} \beta$. The two arms have comparable brightness in the other lines. This is also in line with the results from the Doppler tomography.

To further investigate the properties of the asymmetric arcs we divided the eclipse map into azimuthal slices (i.e., "slices of pizza") and computed the radius at which the intensity is a maximum for each azimuth. A corresponding Keplerian velocity is obtained from the radius of maximum intensity assuming that $M_{1}=1.02 M_{\odot}$ and $R_{L 1}=0.81 R_{\odot}$ for IP Peg (Marsh \& Horne 1990). This exercise allows us to trace the distribution of the spiral structures in radius and azimuth (BHS).

Figure 4 shows the azimuthal intensity distribution $I_{\max }$, radius $R\left(I_{\max }\right)$ and corresponding Keplerian velocity at maximum intensity $v_{\text {kep }}\left[R\left(I_{\max }\right)\right]$. Azimuths are expressed in terms of orbital phase. These are measured from the line joining both stars and increase clockwise for the eclipse maps of Fig. 3. The uncertainties in $I_{\max }, R\left(I_{\max }\right)$ and $v_{\mathrm{kep}}\left[R\left(I_{\max }\right)\right]$ are derived from the Monte Carlo simulations (see Sect. 2.2). Dotted lines in Fig. 4 depict the $1-\sigma$ limits in these distributions.

The two-armed asymmetric structures in the eclipse maps lead to a double-humped shape in the azimuthal intensity distribution. The valleys in the azimuthal intensity distribution trace the region in between the spirals, where $R\left(I_{\max }\right)$ is meaningless. Accordingly, the distributions of $R\left(I_{\max }\right)$ and $v_{\mathrm{kep}}\left[R\left(I_{\max }\right)\right]$ are cut-off in the phase range where the intensity drops below 10 per cent of the peak intensity. The maximum intensity along the outer, blue arm occurs at a radius of $0.55 \pm 0.05 R_{L 1}\left(v_{\mathrm{kep}}=\right.$ $\left.670 \pm 40 \mathrm{~km} \mathrm{~s}^{-1}\right)$, whereas that of the inner, red arm is at $0.30 \pm 0.10 R_{L 1}\left(v_{\mathrm{kep}}=900 \pm 100 \mathrm{~km} \mathrm{~s}^{-1}\right)$. The position (and velocity) of the blue arm is more precisely measured than that of the red arm. The location, radial and azimuthal range of the spiral arms are the same for all lines - from the low-excitation Balmer lines to the high excitation He II and Bowen blend lines - within the uncertainties.

A similar procedure was applied to the Doppler maps of Fig. 3 to compute the observed velocity at the maximum intensity as a function of orbital phase. The results are plotted as solid grey lines in the velocity panels of Fig. 4. The results for 


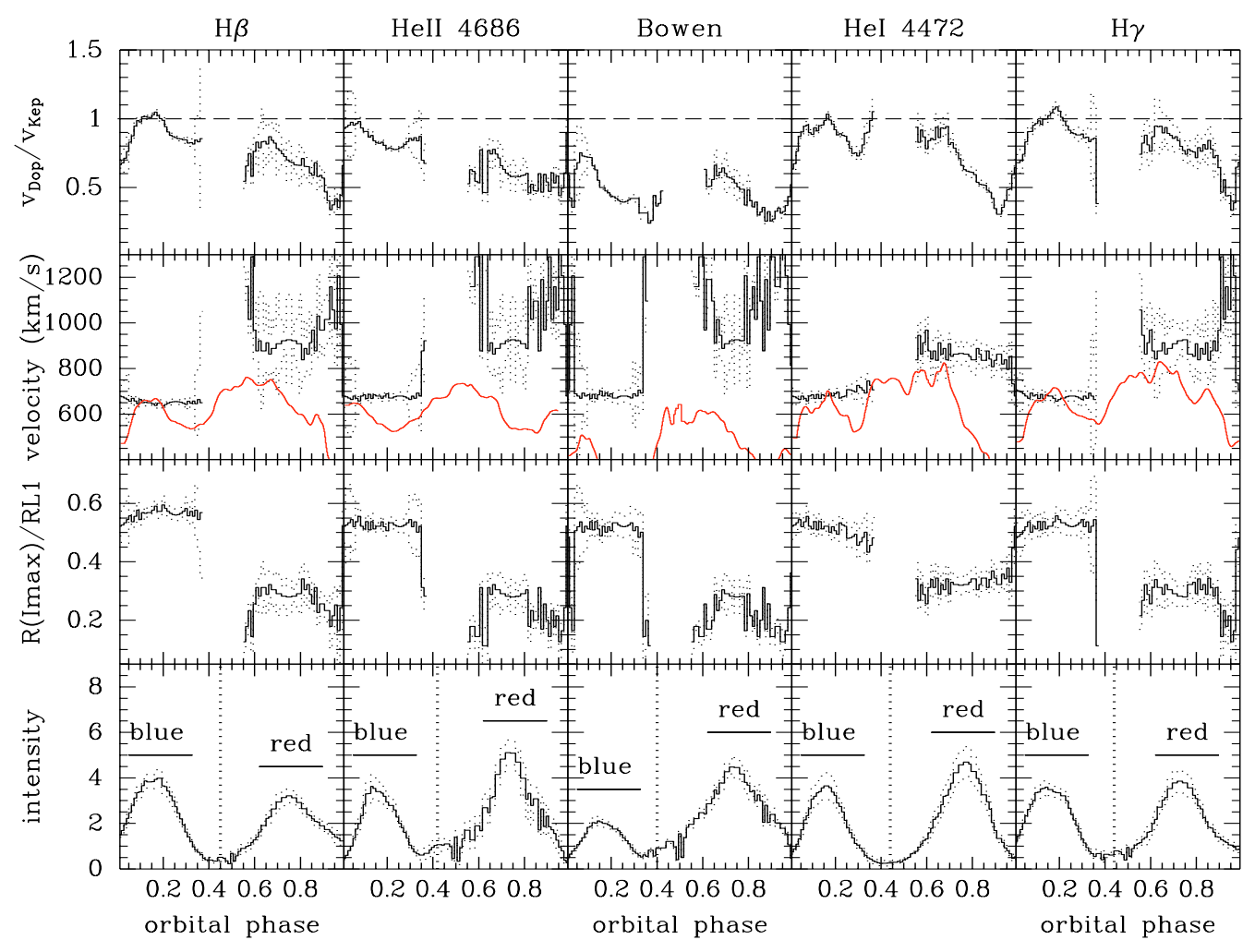

Fig. 4. The dependency with binary phase of the maximum intensity (lowermost panels), radius and corresponding Keplerian velocity at maximum intensity (second and third rows of panels from the bottom) as derived from the line eclipse maps. Dotted lines depict the $\pm 1 \sigma$ limits in these distributions. The velocities were computed assuming $M_{1}=1.02 M_{\odot}$ and $R_{L 1}=0.81 R_{\odot}$ (Marsh \& Horne 1990), and the intensities are plotted in an arbitrary scale. The orbital dependency of the Doppler velocity of maximum intensity (extracted from the Doppler maps of Fig. 3) is shown as a solid grey line in the velocity panels. The location of the spiral arms are indicated in the lowermost panels by horizontal bars labeled "blue" (outer spiral) and "red" (inner spiral). Vertical dotted lines in the lowermost panels mark the phase of minimum intensity of the valley in between the spirals and are used to estimate the spirals opening angle (see Sect. 4). The uppermost panels show the ratio of the observed velocity (Doppler map) to the Keplerian velocity at the same radius (eclipse map). A horizontal dashed line in each panel depicts the reference unity velocity ratio.

the Bowen blend line are less reliable than for the other lines as the corresponding Doppler map is quite noisy (Fig. 3). We shall regard the results of the following analysis for this line as merely illustrative.

The upper panels of Fig. 4 show the ratio of the observed velocity (Doppler map) to the Keplerian velocity at the same radius (eclipse map). A horizontal dashed line in each panel depicts the reference unity velocity ratio. This comparison indicates that the velocity of the emitting gas along the spiral arms is generally lower than the Keplerian velocity at that radius. The difference is small for the blue arm, which shows velocities 10-15 per cent lower than Keplerian. However, the discrepancy is large for the red arm, with differences reaching up to 40 per cent. This indicates that the velocity field along the spiral arms is not Keplerian, in agreement with the results of BHS. This result seems in line with the interpretation of these asymmetric structures as caused by tidally-induced spiral shocks, because the heated (and emitting) post-shock gas is expected to move at velocities lower than the Keplerian velocity of the non-shocked disc orbiting gas at the same radius.

Nevertheless, hydrodynamical simulations by Steeghs \& Stehle (1999) suggest that the departures from a Keplerian flow along the spiral pattern increase with radius, and that one should expect the velocity discrepancy to be larger for the outer, blue arm - just the opposite of what is observed. The larger velocity discrepancy (and possibly also the smaller distance with respect to disc centre) of the red arm may be a consequence of its interaction with the infalling gas stream. In this regard, the hydrodynamical simulations by Bisikalo et al. (1998) and Makita et al. (2000) show that the gas stream may penetrate the disc, forming a bow shock that interacts and mixes with the red arm to enhance emission along a spiral pattern close to the disc centre.

\section{Discussion}

\subsection{Measuring the opening angle of the spirals}

The shape of the azimuthal intensity distribution and the position of maxima/minima intensities are defined by the opening angle of the spiral arms (see the Appendix). For a fixed orientation of the spiral arms (as expected for tidally-induced spirals), the azimuth (binary phase) of maximum/minimum intensity increases as the opening angle of the spiral decreases. We performed simulations with artificial brightness distributions containing spiral arms to calibrate this relation. These are described in the Appendix. It is therefore possible to infer 


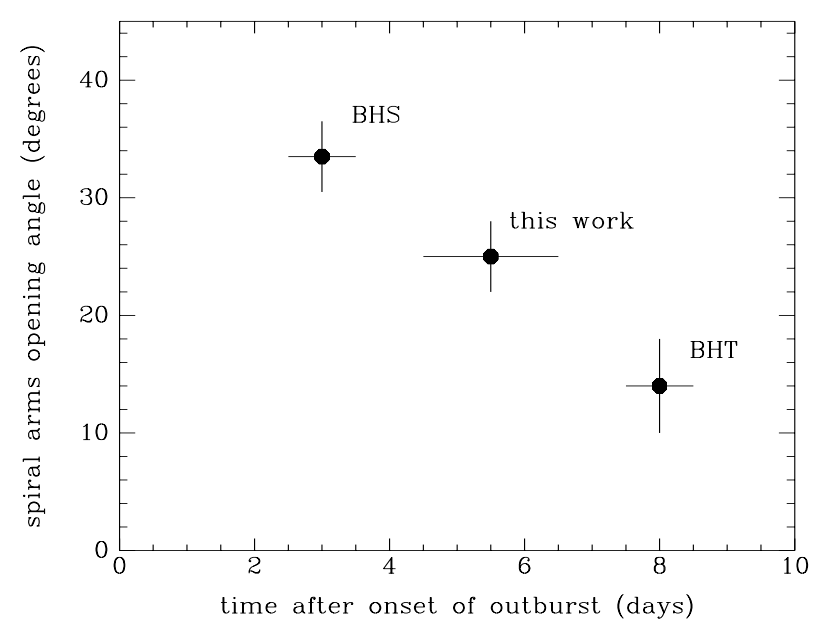

Fig. 5. The measured opening angle of the spiral arms in IP Peg as a function of outburst stage.

the opening angle of the spiral arms from the measured binary phase of minimum in the intensity distribution of Fig. 4.

We fitted a parabola to the lower portion of the $I_{\max }$ distribution to find an average orbital phase of minimum of $0.43 \pm$ 0.02 cycles. From this measurement, we estimate the opening angle of the spiral arms in our data to be $\phi=25^{\circ} \pm 3^{\circ}$. We performed similar analysis with the maximum intensity distributions of BHS and BHT to find opening angles of $\phi=34^{\circ} \pm 3^{\circ}$ and $\phi=14^{\circ} \pm 4^{\circ}$, respectively. Our data were collected 5-6 days after the onset of the 1994 October outburst. Those of BHS and BHT were obtained, respectively, 3 and 9 days after the start of the corresponding outbursts.

Figure 5 shows the evolution of the measured opening angle of the spiral arms in IP Peg with the time from the start of the outburst. There is a clear correlation between the measured spiral opening angle and the stage of the outburst, in the sense that the later the outburst stage (fainter, cooler and smaller accretion disc), the more tightly wound the spiral arms are. This is supported by 2-D and 3-D hydrodynamical simulations of accretion discs, which indicate that the opening angle of the spiral arms is correlated with the disc temperature, with cooler discs showing smaller opening angles (e.g., Makita et al. 2000; Sato et al. 2003).

\subsection{Spiral structures: shocks or irradiation effects?}

The spiral structures seen in IP Peg and a few other outbursting dwarf novae have usually been interpreted as an observational confirmation of tidally induced spiral shocks in accretion discs (Steeghs 2001, and references therein). Smak (2001), and Ogilvie (2002) proposed that these spiral structures are not evidence of spiral shocks, but are caused by irradiation of tidally thickened sectors of the outer disc by the hot, inner disc regions.

Smak's (2001) model predicts that the blue arm is systematically brighter than the red arm. There is no observational support for this claim. Our analysis of 5 different emission lines show that the ratio of the brightness of the two arms is line-dependent and probably also time dependent. For example, both arms have comparable brightness in the He II $\lambda 4686$ Doppler tomogram of the first night of observations, whereas the blue arm appears stronger than the red arm in the Doppler tomogram of the same line in the second night of observations (see Fig. 4 of Morales-Rueda et al. 2000). Our data show no clear evidence that one arm is systematically brighter than the other one.

Our results may be used to set further constraints on both interpretations.

First, the eclipse mapping procedure projects any emission produced at height $z$ above the disc back along the inclined line of sight to the point where it pierces the disc plane, at a distance $z \tan i$ away from the secondary star with respect to its true position (Baptista et al. 1995). Conversely, if the spiral structures are produced in vertically-extended disc regions, their true positions would be at a distance $z \tan i$ towards the secondary star. For the high inclination of IP Peg, even a small vertical height $z=0.03 R_{L 1} \simeq 1.7 \times 10^{9} \mathrm{~cm}$ moves the spiral arms by $\Delta x \simeq 0.2 R_{L 1}$ towards the secondary star with respect to their positions in the eclipse maps. This displacement is enough to shift both arms to the front side of the disc (the disc hemisphere closest to the secondary star). However, the effects raised by the tides from the secondary star are point-symmetric with respect to the white dwarf, for both the shocks and the irradiation interpretations (Steeghs \& Stehle 1999; Makita et al. 2000; Smak 2001; Ogilvie 2002). It would be hard to explain the resulting highly asymmetric geometrical configuration within the framework of tides induced by the secondary star.

In order to avoid completely braking the point-symmetry of the arms with respect to the white dwarf, they must be at heights lower than $z \simeq 0.015 R_{L 1} \simeq 8 \times 10^{8} \mathrm{~cm}$. But this is of the order of (or even lower than) the expected thickness of the accretion disc at the radial position of the arms ${ }^{2}$. Thus, our results suggest that the site of the line emission along the spirals cannot be too far above the orbital plane. There is no evidence of a measurable disc thickening effect.

Second, a consequence of the irradiation model is that mainly the side of the thickened disc regions facing the hot inner disc would be irradiated. When the thickened disc structure is viewed from a highly inclined line of sight towards the secondary star (i.e., around eclipse) the illuminated red arm would be seen mostly face-on, but the blue arm would suffer self-occultation (or would be seen with a much lower effective area). Thus, in the irradiation model one expects that the red arm appears systematically (and possibly significantly) brighter than the blue arm around eclipse (and this brightness difference would be transported to any eclipse map). Eclipse mapping results offer no support for this prediction.

Finally, while sub-Keplerian velocities along the spiral pattern are expected in the spiral shock model, there is no immediate explanation for them in the irradiation model.

Therefore, the combined results from the Doppler tomograms and eclipse maps seem to favour the spiral shock interpretation of the observed asymmetric structures.

\footnotetext{
${ }^{2}$ For a disc opening angle $\alpha \simeq 2^{\circ}-3^{\circ}$, the disc half thickness $H$ at a distance $R \simeq 0.4 R_{L 1}$ from its centre will be $H=R \tan \alpha \simeq$ $(0.014-0.02) R_{L 1} \simeq(0.8-1.1) \times 10^{9} \mathrm{~cm}$.
} 


\section{Conclusions}

We reanalyzed time-resolved spectroscopy of IP Pegasi in outburst with eclipse mapping techniques in order to investigate the location and geometry of the observed spiral structures. We were able to obtain an improved view of the spiral arms with the aid of light curves extracted in velocity bins matching the observed range of velocities of the spiral arms and a double default map tailored for reconstruction of asymmetric structures.

Two-armed spiral structures are clearly seen in all eclipse maps. The arms are located at different distances from the disc centre. The "blue" arm is farther out in the disc $(R=$ $\left.0.55 \pm 0.05 R_{L 1}\right)$ than the "red" $\operatorname{arm}\left(R=0.30 \pm 0.10 R_{L 1}\right)$. The observed difference in radius is significant at the 3- $\sigma$ level. The "red" arm is stronger in the Bowen blend, whereas the "blue" arm is stronger in $\mathrm{H} \beta$. The two arms have comparable brightnesses in the other lines. The brightness ratios derived from the eclipse maps and Doppler tomograms are consistent with each other.

There is evidence that the velocity of the emitting gas along the spiral pattern is lower than the Keplerian velocity for the same disc radius. The discrepancy is lower in the "blue", outer arm - with the measured Doppler velocities being typically 10-15 per cent lower than Keplerian - but is more significant in the "red", inner arm - where the observed velocities can be 40 per cent lower than the Keplerian velocities.

It is possible to measure the opening angle $\phi$ of the spirals from the azimuthal intensity distribution of the eclipse maps. For the data of this paper we measure $\phi=25^{\circ} \pm 3^{\circ}$. We performed a similar analysis with the data of BHS and BHT to find opening angles of $\phi=34^{\circ} \pm 3^{\circ}$ and $\phi=14^{\circ} \pm 4^{\circ}$, respectively. The difference between the measured opening angle of the spirals in these three cases is statistically significant at the 3- $\sigma$ confidence level. There is clear evidence that the opening angle of the spiral arms in IP Peg decreases towards the later stages of the outburst while the accretion disc cools and shrinks. This fits nicely into the expected evolution of a tidally driven spiral wave and it is supported by hydrodynamical simulations of accretion discs.

The sub-Keplerian velocities along the spiral pattern and the clear correlation between the opening angle of the spirals and the outburst stage favors the interpretation of these asymmetric structures as tidally-induced spiral shocks.

It would be crucial to confirm these results with further time-lapse, combined Doppler tomography and eclipse mapping of emission lines of IP Peg along the same outburst cycle.

Acknowledgements. We dedicate this paper to the memory of our colleague and dear friend Emilios Harlaftis. The INT Telescope is operated on the Island of La Palma by the Isaac Newton Group in the Spanish Observatory del Roque de los Muchachos of the Instituto de Astrofisica de Canarias. This work was partially supported by $\mathrm{CNPq} / \mathrm{Brazil}$ through the research grant $62.0053 / 01-1$ - PADCT III/Milenio. R.B. acknowledges financial support from CNPq/Brazil through grants 300.354/96-7 and 301.442/2004-5. L.M.R. was supported by a PPARC post-doctoral grant and by NWO-VIDI grant 639.042.201 to P. J. Groot during the course of this research. T.R.M. acknowledges the support of a PPARC Senior Research Fellowship. DS acknowledges a Smithsonian Astrophysical Observatory Clay Fellowship.

\section{Appendix A: A double default eclipse mapping}

The default map defines the point-spread-function of the reconstructed eclipse map. The original default map of Horne (1985) blurs any asymmetric point-like source into a ring at the same disc radius and is well suited to recover the radial profile of broad and fairly symmetrical disc brightness distributions.

The limited azimuthal smearing default (Rutten et al. 1992; Baptista et al. 1996) added the capability to recover azimuthal information about asymmetrically located light sources such as the bright spot at the disc rim. Nevertheless, it was not designed for reconstructions of highly asymmetric light sources such as the spiral structures seen in the accretion disc of IP Peg in outburst. The azimuthal smearing default map blurs the spiral pattern into a "butterfly"-shaped structure (Harlaftis et al. 2004). It is possible to use these maps to define the range of radii and azimuths covered by the spirals, but it is not possible to trace the spiral pattern - i.e., to measure the spiral opening angle $\phi$.

Harlaftis et al. (2004) suggest the use of a default map which steers the eclipse mapping solution towards a spiral shape in order to improve the reconstruction of spiral structures in IP Peg. The idea is to minimize the azimuthal blur effect, allowing to trace the observed spiral arms.

We followed this idea and created a spiral default map, which blurs the intensity of each point source in the eclipse map along a spiral pattern in the disc. The first problem with this approach is that the spiral default map introduces an extra, ad hoc parameter, namely, the opening angle of the spiral pattern $\phi$. Since the opening angle is not known in advance (on the contrary, this is one of the quantities we wish to measure from the eclipse map), a plausible approach would be to make reconstructions for a set of values of $\phi$ and try to determine which map is the correct one.

In order to test the ability of the eclipse mapping method in recovering the proper value of $\phi$ using the spiral default map, we created artificial maps with two-armed logarithmic spiral patterns for different values of $\phi$. The spirals have a radial width of $0.08 R_{L 1}$ and a radial range between $0.2-0.6 R_{L 1}$ with a brightness cut off $\exp \left[-\left|\left(r-r_{0}\right) / \mathrm{d} r\right|^{3}\right]$ law. The trace of the spirals is defined by,

$\ln r(\theta)=\ln r\left(\theta_{0}\right)+\tan \phi\left(\theta-\theta_{0}\right)$,

where $r$ and $\theta$ are, respectively, the radial and azimuthal coordinates, $\left[\theta_{0}, r\left(\theta_{0}\right)\right]$ defines a reference point along the spiral pattern, and $\phi$ is the spiral opening angle.

We simulated the eclipse of the artificial maps using the geometry of IP Peg $\left(i=81^{\circ}\right.$ and $q=0.5$, Wood \& Crawford $1986)$ to create light curves with added Gaussian noise and obtained reconstructions using spiral default maps for a set of $\phi$ values. These simulations show that there is no correlation between the map with the correct $\phi_{\text {real }}$ and the map of highest entropy in a sequence of reconstructions with a range of values of $\phi$. We further tried to see if one could recover the correct value $\phi_{\text {real }}$ by using a spiral default map with a fixed, arbitrary value $\phi_{0}$. In all cases the asymmetric arcs in the reconstruction are steered towards $\phi_{0}$ instead of towards $\phi_{\text {real }}$. The conclusion is that using a spiral default map is not useful in investigating 
spiral structures in eclipse maps, unless we know in advance the opening angle of the spirals.

With the usual default map of limited azimuthal smearing, the ability to trace the spiral pattern is hampered by the azimuthal smearing effect, which is responsible for the "butterfly" shape of the reconstructed spiral arms (Harlaftis et al. 2004). For an azimuthal gaussian blur width of $\Delta \theta=30^{\circ}$, all point sources in the eclipse map will be azimuthally smeared by $\simeq 90^{\circ}$. An obvious way to minimize the azimuthal smearing effect and improve the reconstruction of asymmetric structures would be to reduce $\Delta \theta$. This corresponds to relaxing the fundamental constraint that the disc brightness distribution has to be the most axi-symmetric as possible. However, eclipse maps obtained with lower values of $\Delta \theta$ start to show criss-crossed arcs at the position of bright compact sources. The reason it that, without the axi-symmetry constraint, the intensity of a compact source is distributed along the arcs that outline the shadow of the secondary star at the corresponding ingress/egress phases (e.g., Horne 1985). The need to avoid this physically unreasonable mapping is what prompted Horne (1985) to propose the default map with azimuthal smearing.

A default map which tries to avoid building structure along the ingress/egress arcs of the secondary star would be particularly interesting for the reconstruction of asymmetric sources, because in this case $\Delta \theta$ could be reduced without the above undesired side effect. The approach of multiple default maps of Spruit (1994) is very useful in this regard.

Following the prescription of Spruit (1994), the concept of the default function $D(j)$ can be generalized to the case of multiple functions by,

$D(j)=\mathrm{e}^{\left(\sum_{i} n_{i} \ln D_{i}\right)}=\prod_{i} D_{i}^{n_{i}}, \quad \sum_{i} n_{i}=1$,

where $n_{i}$ is the exponent of the individual default function $D_{i}$. If $n_{i}>0$, the method will steer the intensities $I(j)$ towards the default map $D_{i}$, whereas if $n_{i}<0$ the method with steer the intensities away from the default map $D_{i}$.

Here we implement a default function consisting of two default maps: a standard default map of limited azimuthal smearing $D_{\text {az }}$ with a positive index $n$, and a default map of crisscrossed $\operatorname{arcs} D_{\text {criss }}$ with a negative exponent $(1-n)$,

$D(j)=\left[D_{\mathrm{az}}(j)\right]^{n} \times\left[D_{\text {criss }}(j)\right]^{(1-n)}, \quad n>1$.

This is the equivalent of simultaneously requesting the most axi-symmetric solution with the least amount of structures along the ingress/egress arcs of the secondary star. The default map of criss-crossed arcs is defined by,

$D_{\text {criss }}(j)=\frac{\sum_{k} w_{j k} I_{k}}{\sum_{k} w_{j k}}$

where

$$
\begin{aligned}
w_{j k}= & \exp \left[-\frac{1}{2}\left(\frac{\phi_{\mathrm{i}}(j)-\phi_{\mathrm{i}}(k)}{\Delta \phi}\right)^{2}\right] \\
& +\exp \left[-\frac{1}{2}\left(\frac{\phi_{\mathrm{e}}(j)-\phi_{\mathrm{e}}(k)}{\Delta \phi}\right)^{2}\right],
\end{aligned}
$$

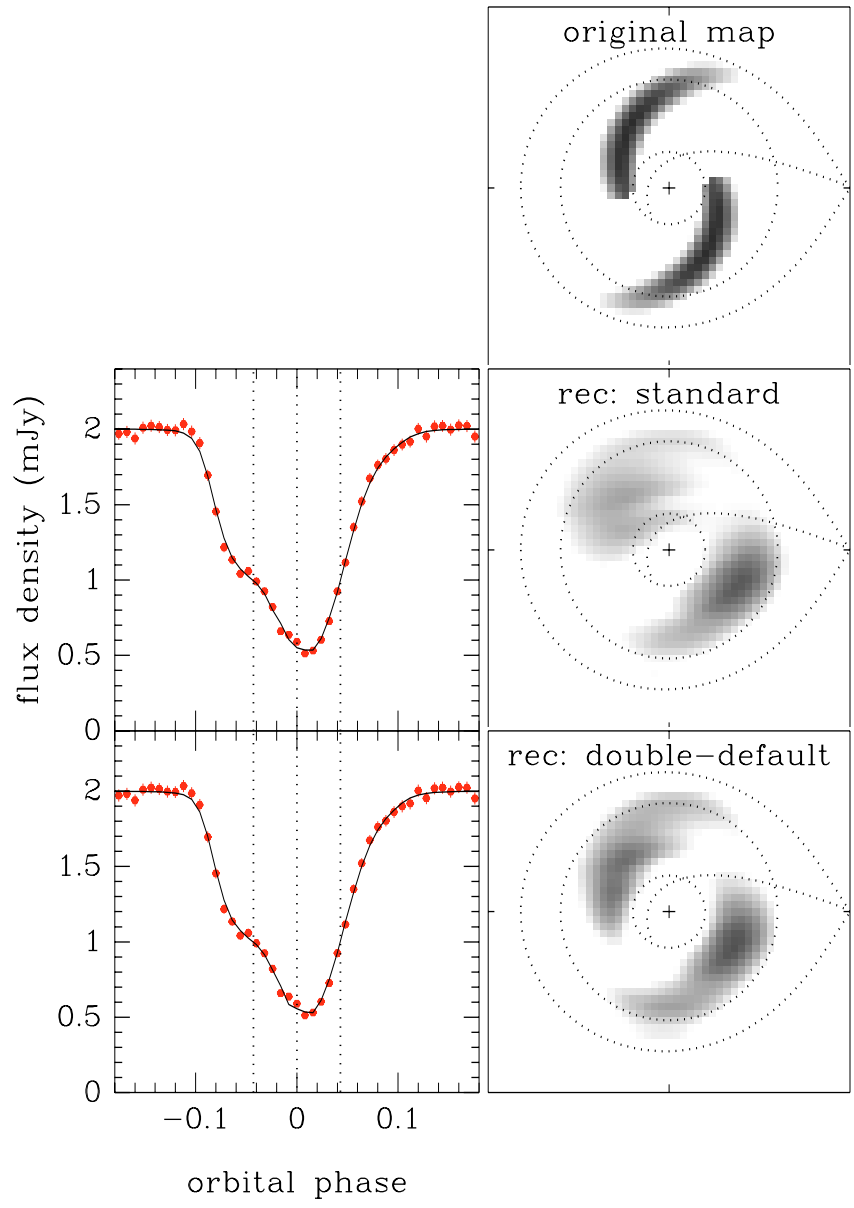

Fig. A.1. Comparison of reconstructions obtained with the standard eclipse mapping (single default map) and the double-default eclipse mapping. The top panel shows the original map with two spiral arms of opening angle $\phi=30^{\circ}$ in a logarithmic greyscale. The left panels depict the simulated data (dots with error bars) and model light curves (solid lines) in each case. Vertical dotted lines indicate the phases of mid-eclipse and of ingress/egress of the disc centre. The right panels show the reconstructions obtained with the standard eclipse mapping (middle panel) and with the double default mapping (lower panel) in the same logarithmic greyscale. The notation is similar to that of Fig. 3.

$I_{k}$ is the intensity of pixel $k, \phi_{\mathrm{i}}(j)$ and $\phi_{\mathrm{e}}(j)$ are the ingress and egress phases of pixel $j$ respectively, and $\Delta \phi$ is the binary phase blur width. Simulations show that good results are obtained for $n \simeq 1.1-1.2$ and $\Delta \phi \simeq 0.002-0.004$ cycles.

This double-default function is tailored for reconstructions of asymmetric brightness distributions. It yields no perceptible improvement in the quality of reconstructions of broad, fairly symmetric brightness distributions found in usual eclipse mapping applications. However, it does make a difference for reconstructions of spiral arms. Figure A.1 compares a reconstruction of a brightness distribution with two spiral arms as obtained with the standard single default map of limited azimuthal smearing with that obtained with the double default map. The two reconstructions were obtained with $\Delta \theta=10^{\circ}$. Because of the reduced $\Delta \theta$ value, a criss-crossed structure develops in the reconstruction with the single default map. It can be seen as a bright arc running across the upper left spiral arm. This arc 


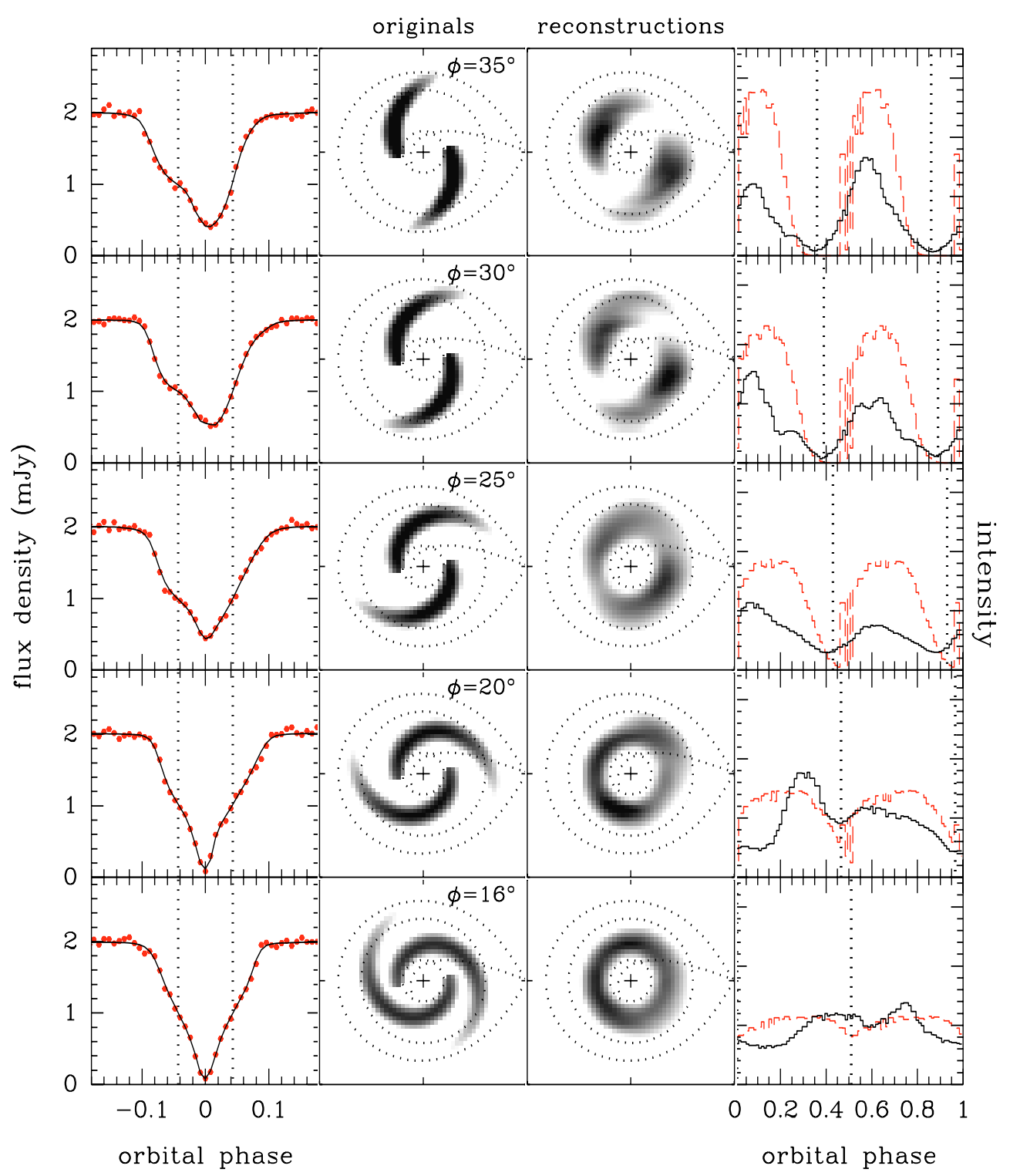

Fig. A.2. Sequence of brightness distributions with spiral arms of different opening angles. Left panel: simulated noisy light curves $(S / N=50$, gray dots with error bars) and eclipse mapping model light curves (solid lines). Vertical dotted lines mark the ingress and egress phases of the disc centre. Middle panels: original maps (left) and eclipse mapping reconstructions with the double-default map (right). The reconstructions were obtained with $\Delta \theta=10^{\circ}$. The notation is similar to that of Fig. A.1. Right panels: the azimuthal dependency of the maximum intensity of the original maps (dashed line) and of the reconstructions (solid line). Vertical dotted lines mark the azimuths of minimum light in each case.

outlines the shadow of the secondary star at the mid-ingress phase of that spiral arm. This artifact is largely suppressed in the reconstruction with the double default map. Furthermore, the "butterfly" shape of the reconstruction with the single default map is replaced by a better defined trace of the spirals in the reconstruction with the double default map.

Figure A.2 shows the performance of the double-default eclipse mapping for the reconstruction of two-armed spiral brightness distributions at different opening angles. The left hand panels display the data and model light curves. It is seen that the shape of the eclipse changes significantly as the opening angle of the spirals is reduced. Original and reconstructed maps are shown in the middle two columns. The right-hand panels show the distributions of the maximum intensity in the map as a function of binary phase (or azimuth, see BHS).
The distribution of the original map is shown as a dashed gray line while that of the reconstruction is indicated by a solid line. Vertical dotted lines mark the azimuths of minimum intensity in each case. Orbital phases are measured with respect to the inner Lagrangian point L1 and increase clockwise.

Wide open spirals $\left(\phi>25^{\circ}\right)$ produce narrow, well defined maxima and minima in the azimuthal intensity distribution. The corresponding eclipse maps show well defined and separated asymmetric arcs with a reasonably good reconstruction of the shape, radial and azimuthal range of the spirals. As the opening angle is reduced, the maxima of the azimuthal intensity distribution become broader while the minima become narrower. Because of the winding up of the spiral arms, both maxima and minima intensities move towards higher orbital phases in a monotonic, fairly linear way. The shape of the azimuthal 


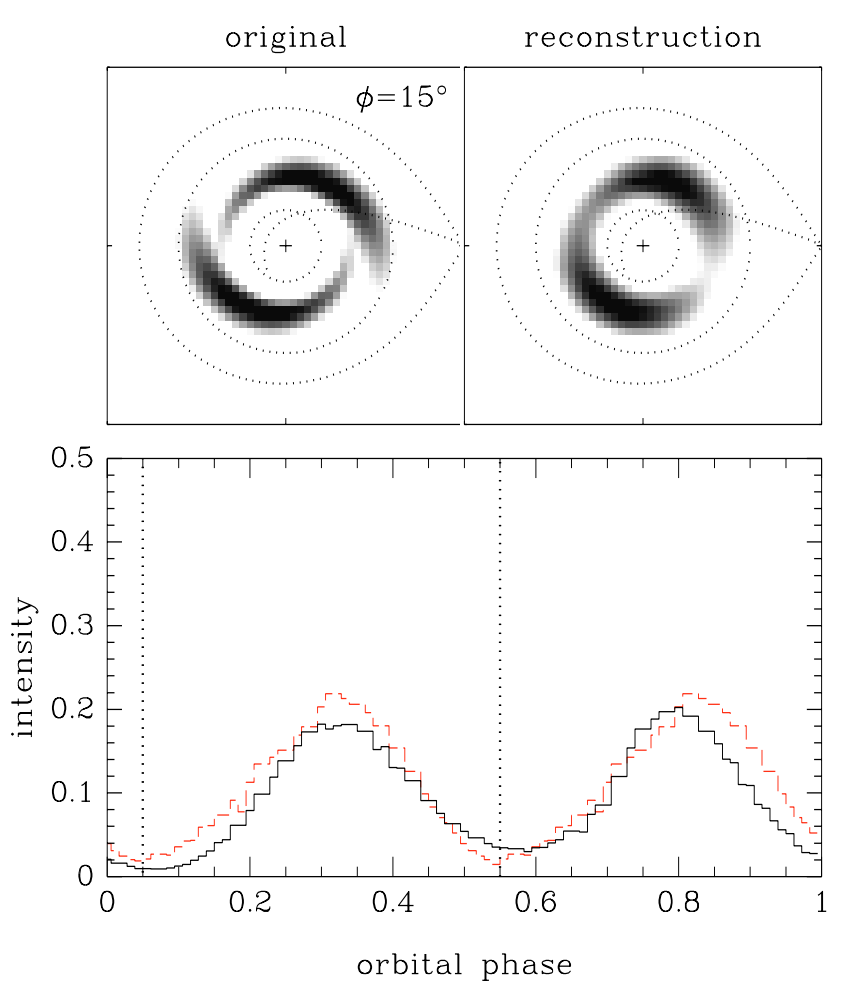

Fig. A.3. Reconstruction of a brightness distribution with spiral arms of $\phi=15^{\circ}$ and radial range $0.3-0.55 R_{L 1}$. Top: original (left) and reconstructed (right) maps. Bottom: the azimuthal dependency of the maximum intensity of the original map (dashed line) and of the reconstruction (solid line). The notation is the same as in Fig. A.2.

distribution and the position of maxima/minima intensities are defined by the opening angle of the spiral arms. Thus, it is possible to estimate the opening angle of the spirals by measuring the position of maxima/minima intensity in the azimuthal distribution.

The maxima of the azimuthal intensity distribution are affected by noise in the light curve and by residual criss-crossed arc effect and are less reliably recovered in the reconstructions. On the other hand, the minima of the azimuthal intensity distribution trace the region in between the spirals. Because there is essentially no flux at these azimuths, their positions are less prone to be distorted by noise. Also, the minima become narrower as the opening angle decreases and are, therefore, easier to measure in the limit of low $\phi$ 's. We therefore choose to measure the opening angle of the spirals from the azimuths of minimum intensity.

For small opening angles the two spiral arms become so tightly wound that they start to overlap in azimuth. Because of the (reduced, but still present) azimuthal smearing effect, they become indistinguishable in the reconstructions and it is no longer possible to use the azimuthal intensity distribution to estimate the opening angle of the spirals. For the simulations presented in Fig. A.2, this occurs at $\phi_{\min } \simeq 16^{\circ}$. The minimum opening angle that is possible to measure depend on the radial extension of the spiral arms. If the spiral pattern does not extend as far inside the disc as those in Fig. A.2 it is possible to measure $\phi$ for spirals with smaller opening angles than the limit of the above example. This is illustrated in Fig. A.3, which shows the results for a two-armed spiral pattern distribution with $\phi=15^{\circ}$ and a reduced radial range of $0.3-0.55 R_{L 1}$. The azimuthal intensity distribution is easily recovered in this case, allowing a precise measurement of the azimuth of minimum intensity. The reconstructed brightness distribution is similar to those of the IP Peg eclipse maps of BHT.

From our simulations we find the following empirical relation between the spiral opening angle $\phi$ and the observed orbital phase of minimum intensity $\Phi_{\text {orb }}\left(I_{\min }\right)$,

$\phi($ degrees $)=\frac{23.25}{\Phi_{\text {orb }}\left(I_{\min }\right)}-29.6$.

Thus, by using a double default map and by measuring the minima of the azimuthal intensity distribution in the resulting eclipse map it is possible to estimate the opening angle $\phi$ of the spiral arms in IP Peg.

\section{References}

Baptista, R., \& Steiner, J. E. 1993, A\&A, 277, 331

Baptista, R., et al. 1994, ASP Conf. Ser., 56, ed. A. Shafter (ASP: San Francisco), 259

Baptista, R., Horne, K., Hilditch, R., Mason, K. O., \& Drew, J. E. 1995, ApJ, 448, 395

Baptista, R., Steiner, J. E., \& Horne, K. 1996, MNRAS, 282, 99

Baptista, R., Harlaftis, E. T., \& Steeghs, D. 2000, MNRAS, 314, 727

Baptista, R., Haswell, C. A., \& Thomas, G. 2002, MNRAS, 334, 198

Bisikalo, D. V., Boyarchuk, A. A., Chechetkin, V. M., Kuznetsov, O. A., \& Molteni, D. 1998, MNRAS, 300, 39

Godon, P., Livio, M., \& Lubow, S. 1998, MNRAS, 295, L11

Harlaftis, E. T. 1999, A\&A, 346, L73

Harlaftis, E. T., Baptista, R., Morales-Rueda, L., Marsh, T. R., \& Steeghs, D. 2004, A\&A, 417, 1063

Harlaftis, E. T., Steeghs, D., Horne K., Martín E., \& Magazzú A. 1999, MNRAS, 306, 348

Horne, K. 1985, MNRAS, 213, 129

Makita, M., Miyawaki, K., \& Matsuda, T. 2000, MNRAS, 316, 906

Marsh, T. R., \& Horne, K. 1988, MNRAS, 235, 269

Marsh, T. R., \& Horne, K. 1990, ApJ, 349, 593

Morales-Rueda, L., Marsh, T. R., \& Billington, I. 2000, MNRAS, 313, 454

Ogilvie, G. I. 2002. MNRAS, 330, 937

Rutten, R. G. M., van Paradijs, J., \& Tinbergen, J. 1992, A\&A, 254, 159

Saito, R., Baptista, R., \& Horne, K. 2005. A\&A, 433, 1085

Sato, J., Sawada, K., \& Ohnishi, N. 2003, MNRAS, 342, 593

Sawada, K., Matsuda, T., \& Hachisu, I. 1986, MNRAS, 219, 75

Smak, J. I. 2001, Acta Astron., 51, 295

Spruit, H. C. 1994, A\&A, 289, 441

Steeghs, D. 2001, Astrotomography, Indirect Imaging Methods in Observational Astronomy, Lecture Notes in Physics (SpringerVerlag), 573, 45

Steeghs, D., Harlaftis, E. T., \& Horne, K. 1997, MNRAS, 290, L28

Steeghs, D., Horne, K., Marsh, T. R., \& Donati, J. F. 1996, MNRAS, 281, 626

Steeghs, D., \& Stehle, R. 1999, MNRAS, 307, 99

Wolf, S., Mantel, K. H., Horne, K., et al. 1993, A\&A, 273, 160

Wood, J. E., \& Crawford, C. S. 1986, MNRAS, 222, 645 